\title{
Real-Time Electron and Hole Transport Dynamics in Halide Perovskite Nanowires
}

\author{
Lisa Janker, ${ }^{\dagger} \|$ Yu Tong, ${ }^{\ddagger}$ Lakshminarayana Polavarapu, $\stackrel{\ddagger}{\dagger} \perp$ Jochen Feldmann, ${ }^{\ddagger}, \|, \perp$

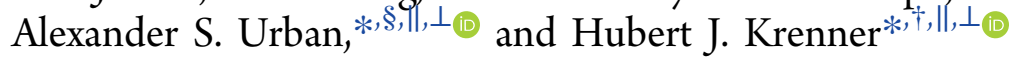

${ }^{\dagger}$ Lehrstuhl für Experimentalphysik 1 and Augsburg Centre for Innovative Technologies, Universität Augsburg, Universitätsstr. 1, 86179 Augsburg, Germany

${ }^{\ddagger}$ Chair for Photonics and Optoelectronics, Nano-Institute Munich, Department of Physics, Ludwig-Maximilians-Universität München, Königinstr. 10, 80539 Munich, Germany

${ }^{\S}$ Nanospectroscopy Group, Nano-Institute Munich, Department of Physics, Ludwig-Maximilians-Universität München, Königinstr. 10, 80539 Munich, Germany

"Nanosystems Initiative Munich (NIM), Schellingstr. 4, 80339 Munich, Germany

${ }^{\perp}$ Center for Nanoscience (CeNS), Ludwig-Maximilians-Universität München, Geschwister-Scholl-Platz 1, 80539 Munich, Germany

\section{Supporting Information}

ABSTRACT: For optoelectronic devices, high transport mobilities of electrons and holes are desirable, which, moreover, should be close to identical. Acousto-optoelectric spectroscopy is employed to probe the spatiotemporal dynamics of both electrons and holes inside $\mathrm{CsPbI}_{3}$ nanowires. These dynamics are induced without the need for electrical contacts simply by the piezoelectric field of a surface acoustic wave. Its radio frequency of $f_{\text {SAW }}=324 \mathrm{MHz}$ natively avoids spurious contributions from ion migration typically occurring

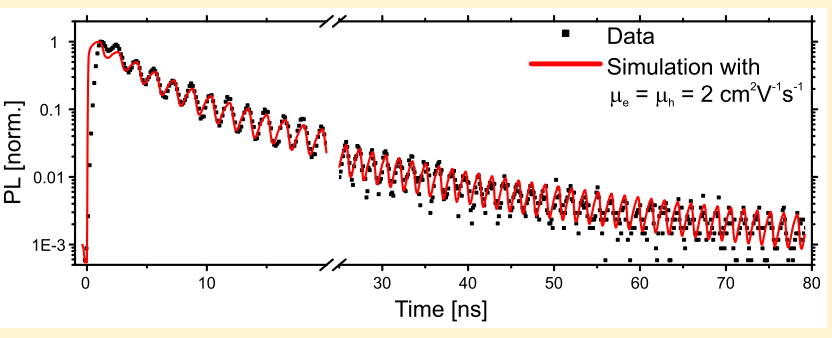
in these materials. The observed dynamic modulation of the photoluminescence is faithfully reproduced by solving the drift and diffusion currents of electrons and holes induced by the surface acoustic wave. These calculations confirm that the mobilities of electrons and holes are equal and quantify them to be $\mu_{\mathrm{e}}$ $=\mu_{\mathrm{h}}=3 \pm 1 \mathrm{~cm}^{2} \mathrm{~V}^{-1} \mathrm{~s}^{-1}$. Additionally, carrier loss due to surface recombination is shown to be largely suppressed in $\mathrm{CsPbI}_{3}$ nanowires. Both findings mark significant advantages over traditional compound semiconductors, in particular, GaAs, for applications in future optoelectronic and photovoltaic devices. The demonstrated sublifetime modulation of the optical emission may find direct application in switchable perovskite light-emitting devices employing mature surface acoustic wave technology.

KEYWORDS: Halide perovskites, nanowires, carrier mobility, surface acoustic waves, dynamic modulation, optical emission dynamics

$\mathrm{T}^{\mathrm{k}}$ he mobility of charge carriers is a key parameter of materials used in optoelectronics. ${ }^{1-6}$ Halide perovskites, an exciting material class, ${ }^{7-11}$ are susceptible to electron-beaminduced degradation and to (halide) ion drift, ${ }^{12}$ limiting suitable measurement techniques. ${ }^{13}$ Studies have thus focused on thin films and single macrocrystals, ${ }^{14}$ with nanostructures proven challenging. However, with opto(electronic) properties varying strongly with size and dimension, ${ }^{15-20}$ these constitute perfect model systems for investigating charge transport. Here we employ contact-free acousto-optoelectric spectroscopy $(\mathrm{AOES})^{21}$ to study the dynamics of photogenerated carriers in $\mathrm{CsPbI}_{3}$ nanowires (NWs) ${ }^{22}$ driven by a surface acoustic wave (SAW). The dynamic, $f_{\text {SAW }}=324 \mathrm{MHz}$, piezoelectric field, which suppresses ion migration, ${ }^{12}$ strongly modulates the optical emission over all times and carrier densities for NWs aligned parallel to the field. Combining these results with numerical simulations, we find the mobilities of electrons and holes to be equal inside individual NWs, with $\mu_{\mathrm{e}}=\mu_{\mathrm{h}}=3 \pm 1$ $\mathrm{cm}^{2} \mathrm{~V}^{-1} \mathrm{~s}^{-1}$. This constitutes a significant advantage over traditional semiconductors like GaAs. Moreover, applications such as high-speed perovskite light sources and advanced wireless sensors are realizable using SAW-based technology. ${ }^{23}$

It is well known that the current flowing in a material is governed by two processes: drift, driven by electric fields, and diffusion, driven by concentration gradients. The efficiencies of both processes are determined by the mobility of the charge carriers in the material. Nanoscale approaches that do not require direct electrical contacts are highly desirable. In this way, such approaches provide direct insight without the need for disentangling, for instance, the impact of Schottky barriers. The electric fields accompanying the propagation of a SAW on a piezoelectric are well-suited to induce carrier transport in

Received: August 19, 2019

Revised: October 10, 2019

Published: October 30, 2019 
a)

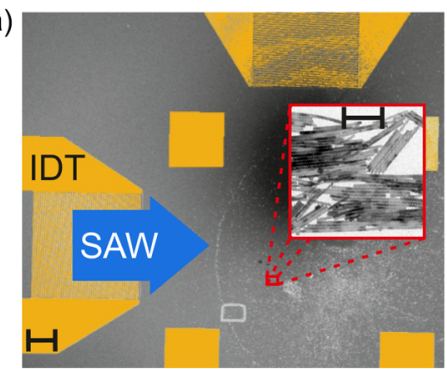

d)

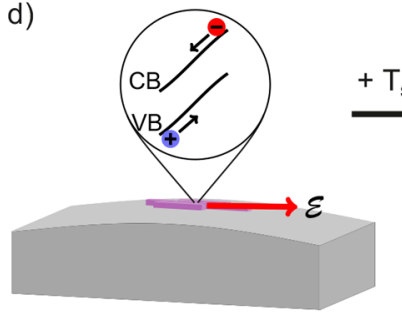

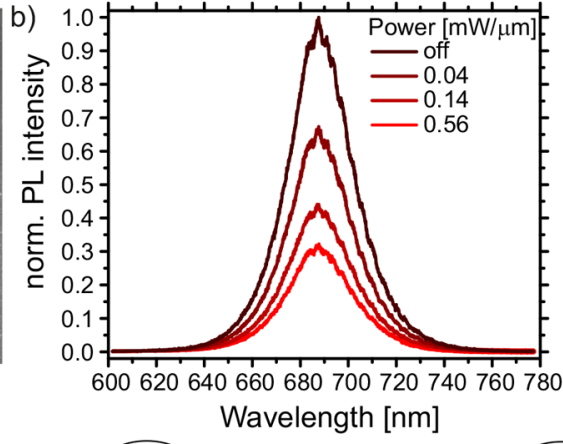

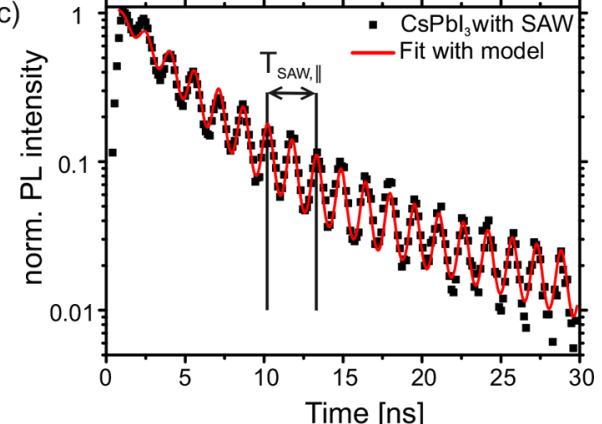
Figure 1. Acousto-optoelectric spectroscopy. (a) Sample design comprising two interdigital transducers (IDTs) to generate perpendicular SAW
beams and individual bundles of aligned $\mathrm{CsPbI}_{3} \mathrm{NWs}$. The scalebar corresponds to $100 \mu \mathrm{m}$. The inset shows a TEM image (scale bar $200 \mathrm{~nm}$ ) of typical $\mathrm{CsPb}_{3} \mathrm{NW}$ bundles. (b) PL spectra of an NW bundle modulated by a $f_{\mathrm{SAW}, \|}=324 \mathrm{MHz}$ SAW of varying intensities and propagating parallel to the long NW axes. As the power density of the SAW increases, the emission is quenched by the piezoelectric field of the SAW. (c) PL decay measurements of $\mathrm{CsPb}_{3} \mathrm{NWs}$ show a pronounced blinking on a sublifetime time scale with a SAW propagating along the NW axis (symbols). The red line shows the best fit of a phenomenological model of time-modulated decay rates. (d) Illustration of the underlying acoustically driven carrier dynamics. Over one acoustic cycle, the electric-field vector gyrates. Every half period, electrons (holes) in the NW are located at stable minima (maxima) or unstable maxima (minima) of the conduction (valence) band. In between, the electric-field vector is parallel to the NW axis, inducing drift motion, which induces a dissociation of photoexcited electron-hole pairs.

nanosystems placed on the surface. ${ }^{24-28}$ We employ this highly versatile AOES to small bundles of aligned $\mathrm{CsPbI}_{3} \mathrm{NWs}$ and determine the ensemble mean of $\mu_{\mathrm{e}}$ and $\mu_{\mathrm{h}}$ inside the NWs at room temperature.

Our experimental setup for AOES is depicted in Figure 1a. Interdigital transducers (IDTs) patterned on a $\mathrm{LiNbO}_{3}$ substrate are used to generate surface acoustic waves simply by applying a radio frequency (rf) voltage. With our chip layout, we are able to generate SAWs propagating horizontally and vertically. $\mathrm{CsPbI}_{3} \mathrm{NWs}$, typically 0.5 to $1 \mu \mathrm{m}$ long and with cross sections of $12 \times 12 \mathrm{~nm}$, are synthesized via solutionbased tip sonication and drop-cast directly on the $\mathrm{LiNbO}_{3}$ surface. (See the Methods section.) ${ }^{22}$ Here they form aligned bundles ( 5 to $10 \mu \mathrm{m}$ long and 1 to $1.5 \mu \mathrm{m}$ wide) at the intersection of the two perpendicular SAW beams. (See the Methods section.) For our experiments, we selected NW bundles aligned parallel to the propagation of one SAW beam and confirmed their coupling to the dynamic piezoelectric field. Figure 1b shows the photoluminescence (PL) spectra of an isolated NW bundle at room temperature under the presence of a $f_{\mathrm{SAW}, \|}=324 \mathrm{MHz}\left(\right.$ period $T_{\mathrm{SAW}, \|}=3.08 \mathrm{~ns}$ ) SAW. As the amplitude of the SAW increases, the PL emission decreases due to a separation of electrons and holes by the SAW's piezoelectric field, as previously observed in III-V semiconductors and nanosystems. ${ }^{21,29-31}$ The dynamic nature of this process is confirmed by measuring the time dependence of the emission signal. In Figure 1c, we show the PL time transient of the NWs with a SAW present (symbols). The transient for the SAW-modulated NW bundle exhibits a pronounced oscillation with a period equal to half of the SAW period: $\frac{1}{2} T_{\mathrm{SAW}, \|}=1.54 \mathrm{~ns}$, which persists over the full duration of the unperturbed transient without the SAW applied (cf. black line in Figure 2a). Importantly, the PL intensity recovers nearly completely compared with the unperturbed case after every full oscillation. The red line is a best fit of a phenomenological model assuming a $\frac{1}{2} T_{\mathrm{SAW}, \|}$ periodic time modulation of the decay rate by the SAW detailed in the Supporting Information. Without an excited SAW, the NWs show a nonexponential, monotonic decay (cf. black line in Figure 2a). PL decay is known to be quite complex in perovskites, with free-carrier and excitonic recombination both occurring and strong inhomogeneity in ensembles leading to subpopulations. ${ }^{32-35}$ To quantify and reproduce the decay dynamics with and without the SAW, we try to fit the original decay as best as possible, which is achieved through a tripleexponential function with time constants $\tau_{0,1}=17.1 \mathrm{~ns}, \tau_{0,2}=$ $5.4 \mathrm{~ns}$, and $\tau_{0,3}=1.8 \mathrm{~ns}$, respectively. The mechanism underlying our experimental findings is depicted in Figure 1d. As the piezoelectric SAW propagates along the NW bundle, the local electric potential is superimposed on the band structure of the NW. At one distinct time during the acoustic cycle, the maximum of the electric potential is located at the position of the NW bundle. This creates an unstable point for electrons in the conduction band (CB) and a stable point for holes in the valence band (VB), respectively. This situation is reversed after $\frac{1}{2} \cdot T_{\mathrm{SAW}, \|}$. In between, the band structure is maximally inclined, and electrons and holes experience a large electric field $(\mathcal{E})$ pointing along the axes of the NWs in the bundle. This oscillating electric field dissociates electron-hole pairs, inducing a drift motion in opposite directions for the two charge carrier species and giving rise to a cyclic motion. At times at which $\mathcal{E}$ is perpendicular to the NW axis, the charge carriers locate spatially, which enhances the radiative 

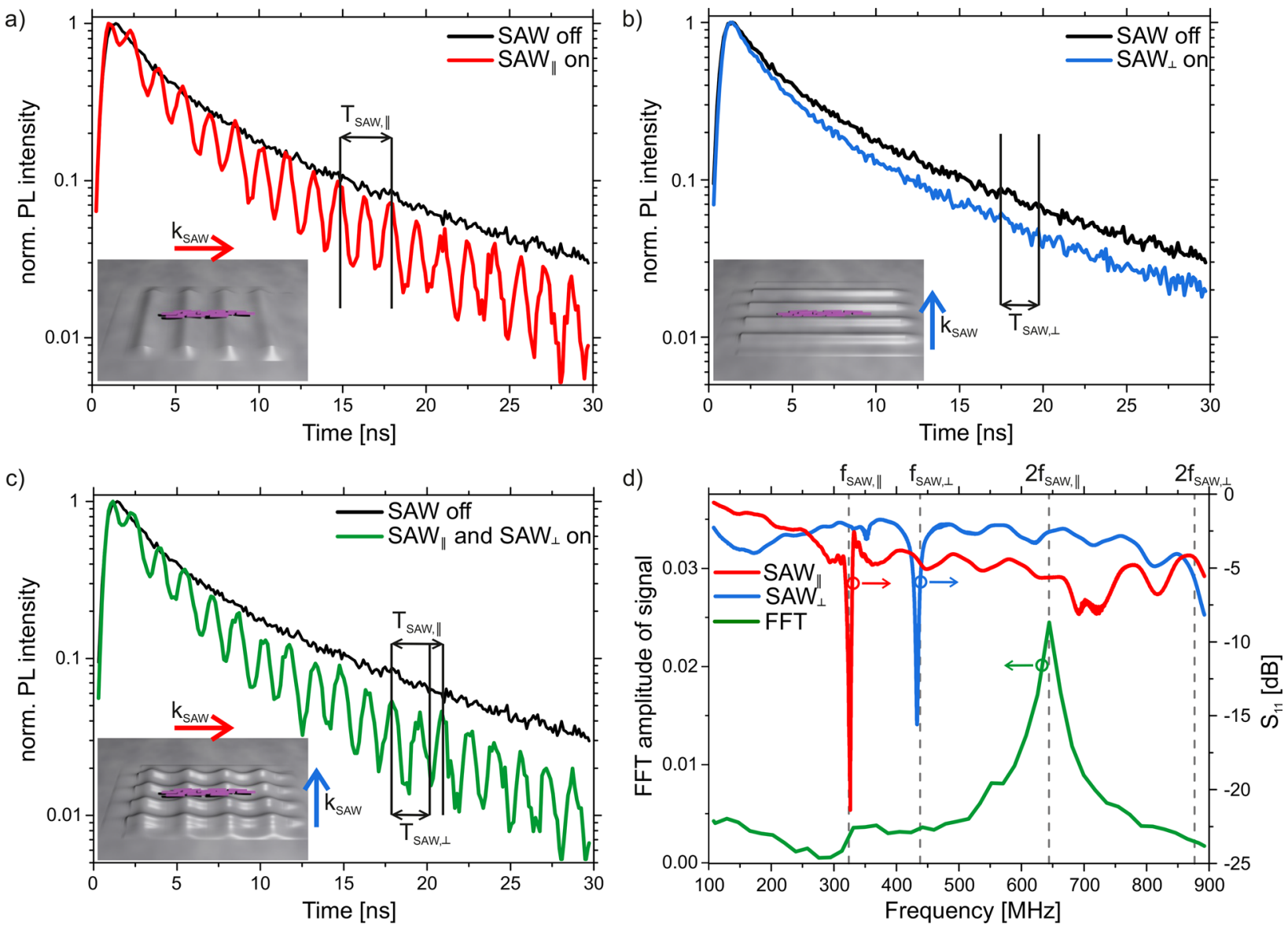

Figure 2. Anisotropy of SAW-modulated PL decay. Time-dependent PL for a SAW applied (a) parallel (red) and (b) perpendicular (blue) to the NW bundle axis compared with a reference measurement without SAW (black lines). The SAW frequencies are $f_{\mathrm{SAW}, \|}=324 \mathrm{MHz}\left(T_{\mathrm{SAW}, \|}=3.08\right.$ ns) and $f_{\mathrm{SAW}, \perp}=438 \mathrm{MHz}\left(T_{\mathrm{SAW}, \perp}=2.28 \mathrm{~ns}\right)$ in panels a and b, respectively. In both cases, an rf power of $P_{\mathrm{rf}}=29 \mathrm{dBm}$ was applied to the IDTs to generate SAWs. The corresponding period of the SAWs is marked in the graphs. (c) Both SAWs simultaneously applied onto the same NW bundle showing no further enhancement of the PL modulation. (d) Fast Fourier transform (FFT) of the modulated signal (green) in panel c and the reflected electrical power of the two IDTs used to excite the parallel (red) and perpendicular (blue) SAW beams. The frequency response clearly only shows a modulation at $2 \cdot f_{\mathrm{SAW}, \|}=648 \mathrm{MHz}$ and no signatures of any other characteristic frequencies.

recombination. This is the reason for the observed $\frac{1}{2} \cdot T_{\mathrm{SAW}, \|}$-periodic intensity modulation. The emission itself is not completely quenched, which indicates that, in fact, not all excitons are dissociated. These observations are in stark difference to analogous experiments on GaAs NWs at cryogenic temperatures. ${ }^{21}$ First, therein the PL is highly quenched and never recovers to the value in the unperturbed case because of highly efficient surface recombination at the end of the GaAs NWs. ${ }^{36}$ Second, complete suppression can be achieved. Third, the modulation occurs with a period equal to the SAW modulation period. In uncapped or surface-passivated GaAs NWs, efficient transfer to the surface does not allow similar experiments at room temperature. On long time scales $>50 \mathrm{~ns}$, the maxima of the modulated PL emission in our $\mathrm{CsPbI}_{3} \mathrm{NWs}$ remain below the unperturbed transient. This indicates a finite, yet weak increase in the nonradiative recombination of dissociated carriers. The incomplete suppression is a direct consequence of the larger exciton binding energy in perovskite semiconductors $\left(E_{\mathrm{B}}=15-25\right.$ $\mathrm{meV}$ for nanoscale $\left.\mathrm{CsPbI}_{3}\right)^{15,33,37}$ compared with GaAs $(4.7$ $\mathrm{meV}$ ) and related $\mathrm{III}-\mathrm{V}$ compounds. ${ }^{38}$ The larger binding energies decrease the ionization rate of the exciton, which depends exponentially on $E_{\mathrm{B}}$ for a given $|\mathcal{E}|$. For materials with even larger binding energies such as $2 \mathrm{D}$ semiconductors like monolayer $\mathrm{MoS}_{2}$, exciton dissociation can be almost completely suppressed. ${ }^{39}$ The halving of the modulation period in comparison with GaAs is a direct fingerprint of the transport mobilities of electrons $\left(\mu_{\mathrm{e}}\right)$ and holes $\left(\mu_{\mathrm{h}}\right)$ being equal. ${ }^{21}$ In this traditional semiconductor, the masses of the two charge carriers are very dissimilar, with $\mu_{\mathrm{h}} \leq 0.1 \mu_{\mathrm{e}}$. Consequentially, holes remain essentially stationary over a large range of $\mathcal{E}$, and the spatial overlap of the charge carriers is restored only once per cycle. In contrast, our observation of a $0.5 \cdot T_{\mathrm{SAW}, \|}$-periodic modulation for $\mathrm{CsPbI}_{3} \mathrm{NWs}$ proves that both carrier species are mobile. It is, in particular, a direct fingerprint that the mobilities are equal $\left(\mu_{\mathrm{e}}=\mu_{\mathrm{h}}\right)$, as commonly assumed for halide perovskite semiconductors.

To confirm that the observed modulation indeed arises from the cyclic drift of electrons and holes along the axes of the individual NWs, we study the dynamic modulation for different SAW propagation directions. In Figure 2, we compare the unmodulated decay (black lines) to that with the SAW propagating parallel and perpendicular to the long NW axes in panels a and $b$, respectively. We observe a clear directionality with no modulation of the emission observable for the perpendicular configuration. Additionally, there is no apparent change in the modulation signal if both parallel and perpendicular SAW beams are simultaneously applied, as 

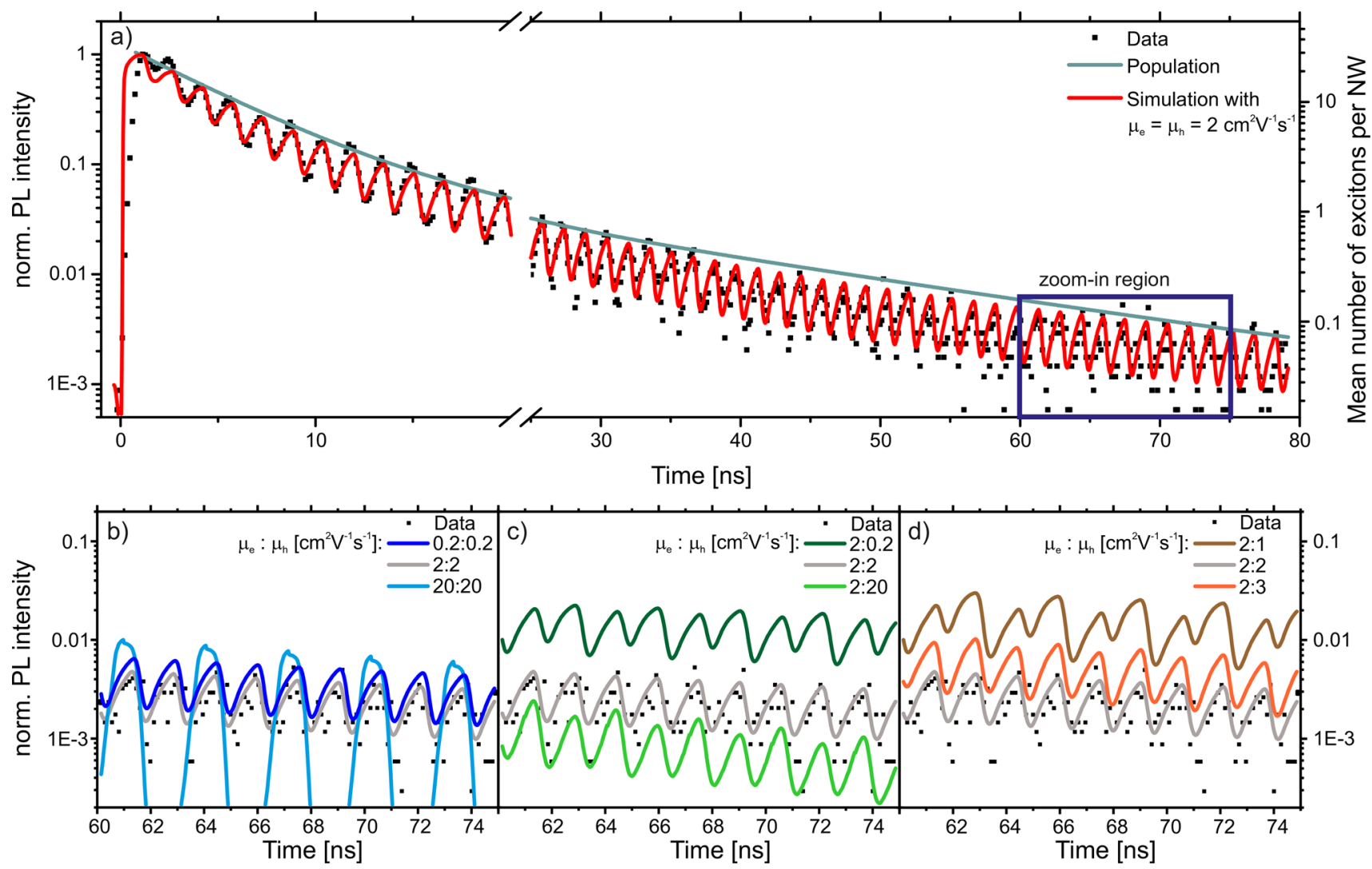

Figure 3. Extracting charge-carrier mobilities from PL modulation. (a) Experimentally observed SAW-modulated PL transient (black symbols). The transient was derived from the calculated SAW-driven spatiotemporal carrier dynamics for $\mu_{\mathrm{e}}=\mu_{\mathrm{h}}=2 \mathrm{~cm}^{2} \mathrm{~V}^{-1} \mathrm{~s}^{-1}$ (red line). The gray line corresponds to the electron-hole pair density per NW. (b-d) Detailed comparison of calculated transients (lines) for different values of $\mu_{\mathrm{e}}$ and $\mu_{\mathrm{h}}$ to the experimental data (symbols). The comparison is performed in the time window indicated by the box in panel a. In panel $b$, the order of magnitude of $\mu_{\mathrm{e}}$ and $\mu_{\mathrm{h}}$ is determined. Panel c assumes mobility ratios similar to that of GaAs, and panel d confirms $\mu_{\mathrm{e}}=\mu_{\mathrm{h}}$.

shown in the time transient in Figure 2c. To confirm this, we further analyze these data in the frequency domain, as depicted in Figure $2 \mathrm{~d}$. The red and blue lines show the electrical power reflected from the transducers used to generate the parallel and perpendicular SAW beams. The two dips at $f_{\mathrm{SAW}, \|}=324 \mathrm{MHz}$ and $f_{\mathrm{SAW}, \perp}=438 \mathrm{MHz}$ correspond to the excitation of the two SAW beams. The Fourier transform of the PL modulation (green line) from Figure 2c exhibits a single peak at $f=648$ $\mathrm{MHz}$, which precisely matches $2 \cdot f_{\mathrm{SAW}, \|}$. No signal is observable at $f=876 \mathrm{MHz}$. These findings prove that electrons and holes are efficiently dissociated along the axes of the NWs, whereas the small cross section of the NWs effectively prevents this process in the perpendicular direction. ${ }^{31}$ The Fourier analysis of all measured transients is presented and discussed in the Supporting Information to further corroborate our data and their interpretation. Additionally, the observed clear directionality shows that (i) no carrier transfer occurs between NWs loosely forming the bundle, and thus (ii) the observed modulations represent an ensemble mean. The latter is important because no statistical analysis of a large number of single NW experiments is required.

Having confirmed that the observed modulation of the optical emission is induced by acoustically driven spatiotemporal drift dynamics of electrons and holes, we can perform a detailed analysis of the actual values of $\mu_{\mathrm{e}}$ and $\mu_{\mathrm{h}}$. To this end, we calculate the modulation of the PL signal by solving the semiclassical drift and diffusion equations, ${ }^{21,40}$ considering the triple exponential decay of the unperturbed NW emission without the SAW applied. The most relevant details of this model are presented in the Supporting Information. Full descriptions can be also found in the literature..$^{21,40-42}$ To model the PL decay under SAW excitation, as previously described, we require only two parameters, the charge-carrier mobilities, $\mu_{\mathrm{e}}$ and $\mu_{\mathrm{h}}$. Figure $3 \mathrm{a}$ shows the experimentally observed (symbols) and calculated (red line) PL intensities as a function of time assuming $\mu_{\mathrm{e}}=\mu_{\mathrm{h}}=2 \mathrm{~cm}^{2} \mathrm{~V}^{-1} \mathrm{~s}^{-1}$. Remarkably, the calculated PL decay reproduces the experimental data over the full duration of PL emission. The gray solid line corresponds to the mean exciton density per NW derived using an empirical model discussed in the Supporting Information. Over the duration of the observed PL decay, this density decreases by more than two orders of magnitude. We substantiate our mobility analysis by computing a series of time transients for different combinations of $\mu_{\mathrm{e}}$ and $\mu_{\mathrm{h}}$, which we compare to the experimental data in the time interval $60 \leq t \leq 75 \mathrm{~ns}$ after photoexcitation, when the differences between the calculated transients are most pronounced. As underpinned by the following detailed comparative study, the simulated time transients are most sensitive to the assumed values of $\mu_{\mathrm{e}}$ and $\mu_{\mathrm{h}}$. In Figure $3 \mathrm{~b}$, we set $\mu_{\mathrm{e}}=\mu_{\mathrm{h}}$ and increase (light-blue line) and decrease (darkblue line) $\mu_{\mathrm{e}}=\mu_{\mathrm{h}}$ by a factor of 10 . Clearly, these calculated transients deviate strongly from the data (symbols). At this point, we have identified the correct orders of magnitude of $\mu_{\mathrm{e}}$ and $\mu_{\mathrm{h}}$ and can examine their ratio in detail. For the simulation shown in Figure $3 \mathrm{c}, \mathrm{d}$, we keep $\mu_{\mathrm{e}}=2 \mathrm{~cm}^{2} \mathrm{~V}^{-1} \mathrm{~s}^{-1}$ fixed. In 
Figure $3 c$, we increase and reduce $\mu_{\mathrm{h}}$ by one order of magnitude to $\mu_{\mathrm{h}}=20 \mathrm{~cm}^{2} \mathrm{~V}^{-1} \mathrm{~s}^{-1}$ (light-green line) and $\mu_{\mathrm{h}}=$ $0.2 \mathrm{~cm}^{2} \mathrm{~V}^{-1} \mathrm{~s}^{-1}$ (dark-green line), respectively. Again, both calculated transients strongly deviate from our experimental finding. Most strikingly, even if we assume only small deviations, $\mu_{\mathrm{h}}=3 \mathrm{~cm}^{2} \mathrm{~V}^{-1} \mathrm{~s}^{-1}$ and $\mu_{\mathrm{h}}=1 \mathrm{~cm}^{2} \mathrm{~V}^{-1} \mathrm{~s}^{-1}$ shown as orange and brown lines in Figure $3 \mathrm{~d}$, respectively, the simulation cannot reproduce the experimental data. Good agreement is found only for equal mobilities, $\mu_{\mathrm{e}}=\mu_{\mathrm{h}}=2 \pm 1$ $\mathrm{cm}^{2} \mathrm{~V}^{-1} \mathrm{~s}^{-1}$. An analogous analysis for a second NW bundle was conducted and is presented in the Supporting Information. Again, we were able to reproduce the data well only for equal hole and electron mobilities with $\mu_{\mathrm{e}}=\mu_{\mathrm{h}}=4 \pm 1 \mathrm{~cm}^{2} \mathrm{~V}^{-1} \mathrm{~s}^{-1}$.

In summary, we employ contact-free AOES to probe chargecarrier transport dynamics inside individual halide perovskite NWs. Because of its dynamic nature and in strong contrast with static electrical methods, this approach enables us to disentangle the rapid dynamics of mobile charge carriers from slow ion migration. Simulations of the underlying drift and diffusion currents reproduce our experimental observations over all time scales of the optical decay. Importantly, this combined study provides direct evidence that the mobilities of electrons and holes are equal in the studied perovskite NWs. Furthermore, we quantify the ensemble means to be $\mu_{\mathrm{e}}=\mu_{\mathrm{h}}=$ $3 \pm 1 \mathrm{~cm}^{2} \mathrm{~V}^{-1} \mathrm{~s}^{-1}$ at room temperature for frequencies in the $300-400 \mathrm{MHz}$ range. Importantly, these values are independent of the carrier concentrations over two orders of magnitude down to the ultralow intrinsic material limit. In addition, they are almost on par and are therefore competitive with those determined for bulk crystals and thin films. ${ }^{14}$ This also confirms that nonradiative surface recombination losses are indeed largely diminished in these materials, which is in strong contrast with traditional semiconductors such as GaAs. ${ }^{36}$ Finally, the 1D geometry of the NWs gives rise to a pronounced directionality, as a carrier transfer between NWs is negligible in the loosely ordered bundles studied here. For highly ordered systems, such as supercrystals assembled from monodisperse nanocrystals, ${ }^{43}$ interparticle hopping processes can occur depending on the spacing of the individual nanocrystals. Our demonstrated contact-free method would be ideally suited to study the resulting hopping mobility for both carrier species systematically, as this is a key figure of merit for the design of nanocrystal- and NW-based optoelectronic and photovoltaic devices. In addition, the acoustically induced modulation of the NW emission provides a promising and versatile route toward the high-speed modulation of perovskite light-emitting devices. Finally, the (photo)conductivity of thin films and 2D systems can be directly probed by SAWs ${ }^{44,45}$ in the electrical domain. Our experimental findings reported here prove that such schemes can indeed be implemented and applied, for instance, in advanced wirelessly interrogable sensors. ${ }^{23}$

Methods. Synthesis of $\mathrm{CsPbl}_{3}$ Nanowires. The $\mathrm{CsPbI}_{3}$ NWs were prepared from $\mathrm{CsPbBr}_{3} \mathrm{NWs}$ by a halide ion exchange reaction, as described in our previous work. ${ }^{22}$ At first, $\mathrm{CsPbBr}_{3} \mathrm{NWs}$ were prepared by an ultrasonication approach. ${ }^{19}$ In a typical synthesis, $0.1 \mathrm{mmol} \mathrm{Cs}_{2} \mathrm{CO}_{3}$ and $0.3 \mathrm{mmol} \mathrm{PbBr}_{2}$ precursor powders were added to a mixture of 1-octadecene $(10 \mathrm{~mL})$, oleylamine $(0.5 \mathrm{~mL})$, and oleic acid $(0.5 \mathrm{~mL})$ and then subjected to tip-sonication at a power of $25 \mathrm{~W}$ for $60 \mathrm{~min}$. The resultant colloidal solution was centrifuged at a speed of $5000 \mathrm{rpm}$ for $10 \mathrm{~min}$; then, the obtained sediment was dispersed in $10 \mathrm{~mL}$ of hexane. This centrifugation process was further repeated twice at a speed of $3000 \mathrm{rpm}$ for $10 \mathrm{~min}$; then, the sediment containing $\mathrm{CsPbBr}_{3} \mathrm{NWs}$ was dispersed in $10 \mathrm{~mL}$ of hexane. To obtain $\mathrm{CsPb}_{3} \mathrm{NWs}$, an excess amount of $\mathrm{PbI}_{2}$ precursor solution (prepared by dissolving $\mathrm{PbI}_{2}(1 \mathrm{mmol})$ in a mixture of $50 \mathrm{~mL}$ of toluene, $1 \mathrm{~mL}$ of oleylamine, and $1 \mathrm{~mL}$ of oleic acid at $100{ }^{\circ} \mathrm{C}$ under continuous stirring) was added to 1 $\mathrm{mL}$ of the as-prepared $\mathrm{Cs} \mathrm{PbBr}_{3} \mathrm{NW}$ colloidal solution under stirring at $40{ }^{\circ} \mathrm{C}$. The completion of the halide exchange was monitored by the PL of the reaction mixture. The resultant solution was centrifuged thrice at $3000 \mathrm{rpm}$ for $10 \mathrm{~min}$; then, the sediment containing $\mathrm{CsPbI}_{3} \mathrm{NWs}$ was dispersed in $1 \mathrm{~mL}$ of hexane.

Acousto-Optoelectric Spectroscopy. SAW chips were fabricated on a Y-cut $\mathrm{LiNbO}_{3}$ substrate. IDTs were defined using standard electron beam lithography and a lift-off process (metallization Ti $5 \mathrm{~nm}, \mathrm{Al} 50 \mathrm{~nm}$ ) to facilitate the excitation of SAWs with $f_{\mathrm{SAW}, \|}=324 \mathrm{MHz}$ and $f_{\mathrm{SAW}, \perp}=438 \mathrm{MHz}$, respectively. Perovskite NWs were drop-casted directly onto the SAW chip without any further surface treatment.

All experiments were performed at room temperature. An externally triggered diode laser emitting $\tau_{\text {laser }} \approx 90$ ps pulses at a wavelength of $532 \mathrm{~nm}$ was focused to a $d_{\text {laser }}=1.5 \mu \mathrm{m}$ spot (probing on average $\sim 150 \mathrm{NWs}$ ) using a $50 \times$ microscope objective to photoexcite carriers. Stroboscopic excitation was realized by actively phase-locking the train of laser pulses to the radio frequency signal generating the SAW. ${ }^{46}$ The duty cycle of the SAW pulses was set sufficiently low to prevent spurious heating of the substrate. ${ }^{47}$ The NW emission was collected by the same objective lens and dispersed in a $0.5 \mathrm{~m}$ imaging grating monochromator. Time-averaged spectra were recorded by a liquid- $\mathrm{N}_{2}$-cooled silicon charge-coupled device. ${ }^{31}$ For time-resolved spectroscopy, the signal was detected by a silicon single photon avalanche detector (SPAD) and recorded by a time-correlated single photon counting module. ${ }^{48}$

\section{ASSOCIATED CONTENT}

\section{Supporting Information}

The Supporting Information is available free of charge on the ACS Publications website at DOI: 10.1021/acs.nanolett.9b03396.

(1) Population decay analysis using a phenomenological model, (2) Fourier analysis of PL transients, (3) model for numerical simulations of spatiotemporal carrier dynamics, and (4) mobility analysis for a second NW bundle (PDF)

\section{AUTHOR INFORMATION}

\section{Corresponding Authors}

*E-mail: hubert.krenner@physik.uni-augsburg.de (H.J.K.).

*E-mail: urban@lmu.de (A.S.U.)

ORCID $\odot$

Lakshminarayana Polavarapu: 0000-0002-9040-5719

Alexander S. Urban: 0000-0001-6168-2509

Hubert J. Krenner: 0000-0002-0696-456X

\section{Author Contributions}

H.J.K. and A.S.U. initiated the project and coordinated the collaboration. H.J.K. designed and supervised the acoustooptoelectric investigations. L.J. conducted the experiments, performed numerical simulations, and analyzed the data, supervised by H.J.K. Y.T. performed the NW synthesis and characterization, supervised by L.P. All authors contributed to 
the interpretation of the data. H.J.K., A.S.U., L.P., and J.F. supervised and inspired the project. H.J.K. and L.J. wrote the manuscript with input from all other authors.

\section{Notes}

The authors declare no competing financial interest.

\section{ACKNOWLEDGMENTS}

We gratefully acknowledge support by the Deutsche Forschungsgemeinschaft (DFG, German Research Foundation) via the German Excellence Initiative's Cluster of Excellence "Nanosystems Initiative Munich" (NIM). The work in Munich was further supported by the Bavarian State Ministry of Science, Research and Arts through the grant "Solar Technologies go Hybrid (SolTech)" and by the DFG under Germany's Excellence Strategy EXC 2089/1390776260. This work was supported by the European Research Council Horizon 2020 through the ERC Grant Agreement PINNACLE (759744). L.J. and H.J.K. thank Achim Wixforth for invaluable discussions and his continuous support.

\section{REFERENCES}

(1) Pearson, G. L.; Bardeen, J. Phys. Rev. 1949, 75 (5), 865-883.

(2) Haynes, J. R.; Shockley, W. Phys. Rev. 1951, 81 (5), 835-843.

(3) Folberth, O. G.; Weiss, H. Z. Naturforsch., A: Phys. Sci. 1955, 10 (8), 615-619.

(4) Chiang, C. K.; Fincher, C. R.; Park, Y. W.; Heeger, A. J.; Shirakawa, H.; Louis, E. J.; Gau, S. C.; MacDiarmid, A. G. Phys. Rev. Lett. 1977, 39 (17), 1098-1101.

(5) Pfeiffer, L.; West, K. W.; Stormer, H. L.; Baldwin, K. W. Appl. Phys. Lett. 1989, 55 (18), 1888.

(6) Parkinson, P.; Joyce, H. J.; Gao, Q.; Tan, H. H.; Zhang, X.; Zou, J.; Jagadish, C.; Herz, L. M.; Johnston, M. B. Nano Lett. 2009, 9 (9), 3349-3353.

(7) Dong, Q.; Fang, Y.; Shao, Y.; Mulligan, P.; Qiu, J.; Cao, L.; Huang, J. Science 2015, 347 (6225), 967-970.

(8) Stranks, S. D.; Snaith, H. J. Nat. Nanotechnol. 2015, 10 (5), 391402.

(9) Kim, Y. C.; Kim, K. H.; Son, D.-Y.; Jeong, D.-N.; Seo, J.-Y.; Choi, Y. S.; Han, I. T.; Lee, S. Y.; Park, N.-G. Nature 2017, 550 (7674), 8791.

(10) Lin, Q.; Armin, A.; Burn, P. L.; Meredith, P. Nat. Photonics 2015, 9 (10), 687-694.

(11) Sutherland, B. R.; Sargent, E. H. Nat. Photonics 2016, 10 (5), 295-302.

(12) Calado, P.; Telford, A. M.; Bryant, D.; Li, X.; Nelson, J.; O’Regan, B. C.; Barnes, P. R. F. Nat. Commun. 2016, 7 (1), 13831.

(13) Birkhold, S. T.; Precht, J. T.; Liu, H.; Giridharagopal, R.; Eperon, G. E.; Schmidt-Mende, L.; Li, X.; Ginger, D. S. ACS Energy Lett. 2018, 3 (6), 1279-1286.

(14) Herz, L. M. ACS Energy Lett. 2017, 2 (7), 1539-1548.

(15) Protesescu, L.; Yakunin, S.; Bodnarchuk, M. I.; Krieg, F.; Caputo, R.; Hendon, C. H.; Yang, R. X.; Walsh, A.; Kovalenko, M. V. Nano Lett. 2015, 15 (6), 3692-3696.

(16) Kovalenko, M. V.; Protesescu, L.; Bodnarchuk, M. I. Science 2017, 358 (6364), 745-750.

(17) Sichert, J. A.; Tong, Y.; Mutz, N.; Vollmer, M.; Fischer, S.; Milowska, K. Z.; García Cortadella, R.; Nickel, B.; Cardenas-Daw, C.; Stolarczyk, J. K.; Urban, A. S.; Feldmann, J. Nano Lett. 2015, 15 (10), 6521-6527.

(18) Tong, Y.; Bladt, E.; Aygüler, M. F.; Manzi, A.; Milowska, K. Z.; Hintermayr, V. A.; Docampo, P.; Bals, S.; Urban, A. S.; Polavarapu, L.; Feldmann, J. Angew. Chem., Int. Ed. 2016, 55 (44), 13887-13892.

(19) Zhu, H.; Fu, Y.; Meng, F.; Wu, X.; Gong, Z.; Ding, Q.; Gustafsson, M. V.; Trinh, M. T.; Jin, S.; Zhu, X.-Y. Nat. Mater. 2015, $14(6), 636-642$.
(20) Akkerman, Q. A.; Rainò, G.; Kovalenko, M. V.; Manna, L. Nat. Mater. 2018, 17 (5), 394-405.

(21) Kinzel, J. B.; Schülein, F. J. R.; Weiß, M.; Janker, L.; Bühler, D. D.; Heigl, M.; Rudolph, D.; Morkötter, S.; Döblinger, M.; Bichler, M.; Abstreiter, G.; Finley, J. J.; Wixforth, A.; Koblmüller, G.; Krenner, H. J. ACS Nano 2016, 10 (5), 4942-4953.

(22) Tong, Y.; Bohn, B. J.; Bladt, E.; Wang, K.; Müller-Buschbaum, P.; Bals, S.; Urban, A. S.; Polavarapu, L.; Feldmann, J. Angew. Chem., Int. Ed. 2017, 56 (44), 13887-13892.

(23) Delsing, P.; Cleland, A. N.; Schuetz, M. J. A.; Knörzer, J.; Giedke, G.; Cirac, J. I.; Srinivasan, K.; Wu, M.; Balram, K. C.; Bäuerle, C.; Meunier, T.; Ford, C. J. B.; Santos, P. V.; Cerda-Méndez, E.; Wang, H.; Krenner, H. J.; Nysten, E. D. S.; Weiß, M.; Nash, G. R.; Thevenard, L.; Gourdon, C.; Rovillain, P.; Marangolo, M.; Duquesne, J.-Y.; Fischerauer, G.; Ruile, W.; Reiner, A.; Paschke, B.; Denysenko, D.; Volkmer, D.; Wixforth, A.; Bruus, H.; Wiklund, M.; Reboud, J.; Cooper, J. M.; Fu, Y.; Brugger, M. S.; Rehfeldt, F.; Westerhausen, C. J. Phys. D: Appl. Phys. 2019, 52 (35), 353001.

(24) Rotter, M.; Rocke, C.; Böhm, S.; Lorke, A.; Wixforth, A.; Ruile, W.; Korte, L. Appl. Phys. Lett. 1997, 70 (16), 2097-2099.

(25) Fuhrmann, D. A.; Krenner, H. J.; Wixforth, A.; Curran, A.; Prior, K. A.; Warburton, R. J.; Ebbecke, J. J. Appl. Phys. 2010, 107 (9), 093717.

(26) Poole, T.; Nash, G. R. J. J. Phys. D: Appl. Phys. 2018, 51 (15), 154001.

(27) Lazić, S.; Chernysheva, E.; Gačević, Ž.; van der Meulen, H. P.; Calleja, E.; Calleja Pardo, J. M. AIP Adv. 2015, 5 (9), 097217.

(28) Hernández-Mínguez, A.; Möller, M.; Breuer, S.; Pfüller, C.; Somaschini, C.; Lazić, S.; Brandt, O.; García-Cristóbal, A.; de Lima, M. M.; Cantarero, A.; Geelhaar, L.; Riechert, H.; Santos, P. V. Nano Lett. 2012, 12 (1), 252-258.

(29) Rocke, C.; Zimmermann, S.; Wixforth, A.; Kotthaus, J. P.; Böhm, G.; Weimann, G. Phys. Rev. Lett. 1997, 78 (21), 4099-4102.

(30) Santos, P. V.; Ramsteiner, M.; Jungnickel, F. Appl. Phys. Lett. 1998, 72 (17), 2099-2101.

(31) Kinzel, J. B.; Rudolph, D.; Bichler, M.; Abstreiter, G.; Finley, J. J.; Koblmüller, G.; Wixforth, A.; Krenner, H. J. Nano Lett. 2011, 11 (4), 1512-1517.

(32) Diroll, B. T.; Nedelcu, G.; Kovalenko, M. V.; Schaller, R. D. Adv. Funct. Mater. 2017, 27 (21), 1606750.

(33) Diroll, B. T.; Zhou, H.; Schaller, R. D. Adv. Funct. Mater. 2018 28 (30), 1800945.

(34) Bohn, B. J.; Tong, Y.; Gramlich, M.; Lai, M. L.; Döblinger, M.; Wang, K.; Hoye, R. L. Z.; Müller-Buschbaum, P.; Stranks, S. D.; Urban, A. S.; Polavarapu, L.; Feldmann, J. Nano Lett. 2018, 18 (8), 5231-5238.

(35) Gabelloni, F.; Biccari, F.; Falsini, N.; Calisi, N.; Caporali, S.; Vinattieri, A. Nanophotonics 2019, 8 (9), 1447-1455.

(36) Demichel, O.; Heiss, M.; Bleuse, J.; Mariette, H.; Fontcuberta i Morral, A. Appl. Phys. Lett. 2010, 97 (20), 201907.

(37) Yang, Z.; Surrente, A.; Galkowski, K.; Miyata, A.; Portugall, O.; Sutton, R. J.; Haghighirad, A. A.; Snaith, H. J.; Maude, D. K.; Plochocka, P.; Nicholas, R. J. ACS Energy Lett. 2017, 2 (7), 16211627.

(38) Adachi, S. J. Appl. Phys. 1985, 58 (3), R1-R29.

(39) Huang, T.; Han, P.; Wang, X.; Ye, J.; Sun, W.; Feng, S.; Zhang, Y. J. Phys. D: Appl. Phys. 2017, 50 (11), 114005.

(40) García-Cristóbal, A.; Cantarero, A.; Alsina, F.; Santos, P. V. Phys. Rev. B: Condens. Matter Mater. Phys. 2004, 69 (20), 205301.

(41) Schülein, F. J. R.; Pustiowski, J.; Müller, K.; Bichler, M.; Koblmüller, G.; Finley, J. J.; Wixforth, A.; Krenner, H. J. JETP Lett. 2012, 95, 575-580.

(42) Schülein, F. J. R.; Müller, K.; Bichler, M.; Koblmüller, G.; Finley, J. J.; Wixforth, A.; Krenner, H. J. Phys. Rev. B: Condens. Matter Mater. Phys. 2013, 88 (8), 085307.

(43) Tong, Y.; Yao, E.-P.; Manzi, A.; Bladt, E.; Wang, K.; Döblinger, M.; Bals, S.; Müller-Buschbaum, P.; Urban, A. S.; Polavarapu, L.; Feldmann, J. Adv. Mater. 2018, 30 (29), 1801117. 
(44) Wixforth, A.; Scriba, J.; Wassermeier, M.; Kotthaus, J.; Weimann, G.; Schlapp, W. Phys. Rev. B: Condens. Matter Mater. Phys. 1989, 40 (11), 7874-7887.

(45) Preciado, E.; Schülein, F. J. R.; Nguyen, A. E.; Barroso, D.; Isarraraz, M.; von Son, G.; Lu, I.-H.; Michailow, W.; Möller, B.; Klee, V.; Mann, J.; Wixforth, A.; Bartels, L.; Krenner, H. J. Nat. Commun. 2015, 6, 8593.

(46) Völk, S.; Knall, F.; Schülein, F. J. R.; Truong, T. A.; Kim, H.; Petroff, P. M.; Wixforth, A.; Krenner, H. J. Appl. Phys. Lett. 2011, 98 (2), 023109.

(47) Weiß, M.; Kapfinger, S.; Reichert, T.; Finley, J. J.; Wixforth, A.; Kaniber, M.; Krenner, H. J. Appl. Phys. Lett. 2016, 109 (3), 033105. (48) Kapfinger, S.; Reichert, T.; Lichtmannecker, S.; Müller, K.; Finley, J. J.; Wixforth, A.; Kaniber, M.; Krenner, H. J. Nat. Commun. 2015, 6 (1), 8540 . 